\title{
A two-choice sound localization procedure for detecting lateralized tinnitus in animals
}

\author{
Henry E. Heffner
}

Published online: 17 March 2011

(C) Psychonomic Society, Inc. 2011

\begin{abstract}
Rats were trained in a two-choice procedure to respond in the direction of left and right sounds. Silent trials, on which no sound was presented and for which the animals received no feedback, were interspersed among the sound trials to determine each animal's natural side preference. Following training, the rats were exposed to a loud tone in the ear opposite their side preference. A shift in responding on the silent trials to the side of the exposed ear indicated that the animals were hearing a sound in that ear (i.e., tinnitus). Simulating lateralized tinnitus by presenting a low-level, continuous sound on one side also caused the rats to shift their responding on the silent trials to that side. Sham exposures indicated that halothane/nitrous oxide anesthesia could reinstate tinnitus in animals that had previously tested positive for it. Exposing rats to loud tones of various frequencies indicated that frequencies near the limits of the rat's hearing range were less likely to cause tinnitus than tones in the midrange.
\end{abstract}

Keywords Tinnitus - Anesthesia - Noise exposure ·

Sensorineural threshold shift · Lateralization · Operant behavior $\cdot$ Rat

Tinnitus refers to the perception of sound in the absence of any corresponding acoustic stimulation; the word itself is from the Latin word for ringing (tinnitus), because the perceived sound is often tonal in nature (see, e.g., Loeb \& Smith, 1967; Martines et al., 2010). Although there are a number of causes of tinnitus, two of the more common ones are exposure to loud sound and the ingestion of large doses

\section{H. E. Heffner $(\square)$}

Department of Psychology, University of Toledo,

2801 West Bancroft,

Toledo, OH 43606, USA

e-mail: hheffne@pop3.utoledo.edu of ototoxic drugs such as quinine or salicylate, the latter being the active ingredient of aspirin (e.g., Bauer \& Brozoski, 2008). In recent years, there has been increased interest in tinnitus, primarily with the goal of finding a treatment for it, although an understanding of how tinnitus arises in the auditory system would also give insight into the neurological basis of the perception of sound. Such studies are best conducted on animals, creating a need for tests that can determine whether an animal has tinnitus.

The usual procedure for assessing animals for tinnitus involves training them on an auditory discrimination task, exposing them to a tinnitus-inducing agent, and testing them to determine whether their performance has changed in some way that suggests they now have tinnitus. The first animal test for tinnitus, developed by Jastreboff, Brennan, Coleman, and Sasaki (1988), involved allowing thirsty animals to drink in the presence of an external sound and training them to stop drinking when the sound is turned off by following sound-off intervals with a foot shock; finding that animals given sodium salicylate or other tinnitusinducing agents are less likely to stop drinking when the external sound is turned off suggests that the animals are hearing sound in the absence of any physical stimulusthat is, they have tinnitus (Jastreboff et al., 1988). Most tests developed since then have also involved training animals to discriminate the presence of external sound from the absence of sound, with the expectation that animals with tinnitus will respond in the absence of external sounds as if a sound were actually present (Bauer, Brozoski, Rojas, Boley, \& Wyder, 1999; Guitton et al., 2003; Guitton \& Dudai, 2007; Heffner \& Harrington, 2002; Kizawa et al., 2010; Lobarinas, Sun, Cushing, \& Salvi, 2004; Rüttiger, Ciuffani, Zenner, \& Knipper, 2003; Zheng, Hooton, Smith, $\&$ Darlington, 2008). Because tinnitus-inducing agents such as loud sounds and ototoxic chemicals usually cause a 
significant hearing loss, all such procedures require controls to demonstrate that a change in an animal's response to sound versus silence is not caused by a reduced ability to hear sound.

Currently, two tinnitus tests differ from the others in the type of discrimination that an animal must make. One is a gap detection test, in which an animal detects a brief silent interval, or gap, in an ongoing sound. If tinnitus similar in frequency to the ongoing sound is present, it is expected to "fill in" the gap, causing a decrement in an animal's gap detection performance (Rybalko \& Syka, 2005; Turner et al., 2006). Any accompanying hearing loss can be controlled either by inducing tinnitus in only one ear or by increasing the intensity of the ongoing sound to ensure that it is clearly audible. Although the idea that ongoing tinnitus could interfere with detecting a temporal gap in an external sound of similar spectral characteristics seems intuitive, this effect has yet to be demonstrated in humans, and the view that tinnitus can interact with the perception of external sounds was questioned many years ago (Davis, Morgan, Hawkins, Galambos, \& Smith, 1950).

The other nontraditional tinnitus test is the two-choice sound localization test (Heffner \& Koay, 2005). In this test, an animal is trained on a sound localization task to make a left or right response to sounds coming from its left or right side, respectively. Silent trials, in which no sound is presented and for which the animal receives no feedback as to how it should respond, are interspersed among the sound trials, and an animal's side preference on those trials is determined. The animal is then exposed to a loud sound in the ear opposite its side preference and tested to see whether it shifts its responding on the silent trials to the side of the exposed ear; doing so would indicate that the animal perceives a sound (tinnitus) that is lateralized to that side, which is conceptually equivalent to a human patient reporting the ear to which their tinnitus is lateralized. In addition to being able to indicate whether an individual animal has lateralized tinnitus, the two-choice procedure would not be expected to be confounded with hearing loss that accompanies exposure to loud sound, an expectation that has been verified by demonstrating that a conductive hearing loss caused by plugging one ear does not cause a shift in responding on silent trials (Heffner \& Koay, 2005). Moreover, because the animals are never given feedback on the silent trials, their responding to their tinnitus may not immediately habituate, making it possible to follow the time course of the tinnitus.

A key assumption of the two-choice test is that exposing an ear to a loud sound will induce tinnitus that is lateralized to that ear-that is, that the tinnitus will neither be lateralized to the unexposed ear nor be bilateral. The three available studies of sound-induced tinnitus in humans suggest that we may make this assumption (Atherley, Hempstock, \& Noble, 1968; Davis et al., 1950; Loeb \&
Smith, 1967). Specifically, each of these studies determined the preexposure absolute thresholds of the subjects, exposed one ear to loud sounds, determined the postexposure absolute thresholds in the exposed ear, and had the subjects match the resulting tinnitus to tones presented to the unexposed ear. None of these studies mentioned the perceived locus of the tinnitus, although it would have been notable had it been lateralized to the unexposed ear. To obtain more information, I contacted Joseph Hawkins, one of the authors and a subject of the Davis et al. study, and he told me that the tinnitus they observed was always lateralized to the exposed ear (J. E. Hawkins, Jr., personal communication, February 25, 2003). Thus, the results of human studies either support or do not conflict with the conclusion that, as long as the exposing sound is presented to only one ear, the resulting tinnitus will be lateralized to that ear.

The purpose of this report is to describe a series of tests that explore the strengths and limitations of the two-choice tinnitus test. These tests include a test for the propensity of sounds of different frequencies to induce tinnitus, a simulated tinnitus test, and a test for the effect of anesthesia on the induction of tinnitus.

\section{Method}

\section{Subjects}

Thirteen male hooded rats (Rattus norvegicus; Harlan Sprague-Dawley) were used in this study. Six of the rats (Rats A-F) were exposed to loud tones and tested for tinnitus; the other 7 were used in an anesthesia control test. The animals were housed in standard solid-bottom cages with grid covers and pelleted corncob bedding ( $1 / 8$-in. pellets; Harlan Teklad). The animals were given free access to rodent chow, and their body weights were measured daily. Water was available only during daily training and testing sessions. Pieces of apple were given as needed to maintain a body weight of approximately $85 \%-90 \%$ ad-lib weight. The use of animals in this study was approved by the University of Toledo Animal Care and Use Committee.

\section{Behavioral apparatus}

Testing was conducted in a carpeted, double-walled sound chamber (IAC Model 1204; Industrial Acoustics, Bronx, $\mathrm{NY} ; 2.55 \times 2.75 \times 2.05 \mathrm{~m}$ ), the walls and ceiling of which were lined with egg-crate foam; the double walls of the sound chamber prevented entry of any sounds audible to the rats. The equipment for behavioral control and stimulus generation was located outside the chamber, and the animals were observed over closed-circuit television. The chamber was carefully checked for the presence of 
extraneous sounds, including frequencies above the range of human hearing, using a sound level meter (described below); no extraneous sound was found. In addition, the lights within the chamber were low-wattage bulbs so that they did not have to be dimmed, since reducing the voltage of incandescent lights can cause their filaments to emit sound ("sing"). In short, there were no audible background sounds in the chamber.

The animals were tested in a cage $(28 \mathrm{~cm}$ long $\times 13 \mathrm{~cm}$ wide $\times 16 \mathrm{~cm}$ high) constructed of 1-in. $(2.54-\mathrm{cm})$ wire mesh. The cage was mounted on a table $1 \mathrm{~m}$ above the chamber floor. Three water spouts (rat water bottle sipper tubes) served as the response manipulanda. The spouts were mounted in a horizontal row at the front of the cage, $3 \mathrm{~cm}$ apart and $6 \mathrm{~cm}$ above the cage floor. Each spout, which had an LED mounted on it $3 \mathrm{~cm}$ back from the tip, was connected to a separate lick circuit. A 2-cm water bowl was mounted $3 \mathrm{~cm}$ above the cage floor below the center spout and was connected to a syringe pump (NE 1000, New Era, Wantagh, NY), located outside the sound chamber, with the water feeding up through a hole in the bottom of the bowl. The water reward was not delivered through the left and right water spouts, because it is difficult to dispense identical amounts, and any imbalance would bias an animal's responding to the spout that delivered more water. Water spouts were used as response manipulanda because a thirsty animal will learn very quickly to lick a spout for water delivered into a water bowl by initially placing water in both the spout and the water bowl. Electric shock was provided by a Coulbourn AC-resistive small animal shocker connected between the side water spouts and the cage floor.

\section{Acoustical apparatus}

Sine waves were generated by a tone generator (Model 2400; Krohn-Hite, Avon, MA) and broadband noise by a noise generator (Model S81-02; Coulbourn, Lehigh Valley, PA). The signals were amplified (Coulbourn Model S82-24) and sent to one of two piezoelectric tweeters (Model KSN 1005A; Motorola, Chicago, IL) that were located just outside the cage, $90^{\circ}$ to the left and right of an animal's head when it was licking the center spout. The training stimulus was broadband noise ( $60 \mathrm{~dB}$ sound pressure level [SPL], $43 \mathrm{~dB}$ above the average threshold of 3 of the rats used in this study) combined with a tone; the noise was used because it is easily localized, and tones were used because it was expected that tinnitus induced by exposure to a loud tone would be tonal (Davis et al., 1950). To accustom the rats to respond to a range of tonal stimuli, the frequency of the tone was changed each day on a random schedule among six different frequencies $(10,12.5,16,20$, 25 , and $32 \mathrm{kHz}$ presented $43-58 \mathrm{~dB}$ above the average rat threshold; Heffner, Heffner, Contos, \& Ott, 1994). Noise alone was presented after an animal had been exposed to a loud sound to induce tinnitus. Because the tone exposures often caused a temporary threshold shift, the intensity of the broadband noise was increased by $5-15 \mathrm{~dB}$, as needed, to maintain good performance on the sound trials.

A simulated tinnitus test was conducted by placing a third loudspeaker at $90^{\circ}$ to one side and presenting a $16-\mathrm{kHz}, 1 / 3$-octave band of noise filtered with a Krohn-Hite 3202 bandpass filter ( $24 \mathrm{~dB} /$ octave roll-off) $25 \mathrm{~dB}$ above the rat's absolute threshold. Noise was used because it is more easily localized by rats than tones (Wesolek, Koay, Heffner, \& Heffner, 2010); it was filtered to make it distinguishable from the broadband noise used in the sound trials.

The SPL was measured using a Brüel \& Kjær (B\&K) 1/4-in (0.64-cm) microphone (Model 4135; B\&K, Naerum, Denmark) and measuring amplifier (B\&K Model 2608). The measuring equipment was calibrated with a pistonphone (B\&K Model 4230).

\section{Behavioral procedure}

A rat was first trained to lick the center spout, an action that turned off the LED on that spout and that was rewarded by delivering water into the water bowl located below it. Next, the rat was trained to lick the center spout and then a side spout to get the water reward. Initially, the correct side response was indicated by presenting the noise/tone from the loudspeaker on that side and by illuminating the LED over the correct spout. Once the rat's performance reached $80 \%$ correct or better, both side LEDs came on to signal that a side response should now be made, without indicating the correct side. Thus, licking the center spout turned off the center LED, illuminated both side LEDs, and turned on the noise + tone signal from either the left or the right loudspeaker. Licking a side spout turned off the side LEDs, and correct responses were rewarded by dispensing $0.06 \mathrm{ml}$ of water into the water bowl. Incorrect responses, on the other hand, were punished with an electric shock delivered between the spout and the cage floor; the shock levels were $1.25 \mathrm{~mA}$ or less and were sufficiently low that the animals never developed a fear of the spouts and continued to respond after receiving a shock. The center LED was then turned back on and the animal permitted to initiate another trial. The frequency of the tone embedded in the noise was changed from session to session to accustom the animals to respond to different tones, on the expectation that their tinnitus would be tonal.

In the final stage of training, "silent" trials were randomly inserted, on which licking the center spout initiated a trial (signaled by the side LEDs), but no sound was presented. The rat was required to make a side 
response, but was neither rewarded with water nor punished with a shock; thus, it received no feedback on the silent trials. Approximately $24 \%$ of the trials in a session were silent trials. At this time, a 50\% feedback schedule was also instituted for the sound trials, according to which, randomly, half of the sound trials were also not followed by water reward or shock, regardless of a rat's side response. Instead, the side LEDs were turned off and the center LED turned back on, so that the rat could start another trial. The purpose of the $50 \%$ feedback schedule was to accustom the rats to a partial-reward schedule so they would be less likely to learn that silent trials were never rewarded or punished. The use of the partial feedback did not affect performance on the sound trials, and responses on silent trials were generally stable.

The left-right trial sequence was determined by a quasirandom schedule (Gellermann, 1933). A correction procedure was used for those sound trials on which a rat made an error and received a shock (i.e., errors on feedback trials), in that the correct side did not change on the next trial; the results of these correction trials were not included in an animal's score. The animals were trained until they consistently performed at a level of $90 \%$ correct or better on the sound trials. The final test consisted of exposing one ear to a loud tone and then testing the rat to determine whether its responding on silent trials shifted to the side of the exposed ear. Each session lasted until the rat had received sufficient water to maintain a consistent body weight, approximately $85 \%-90 \%$ of ad-lib weight, yet still be sufficiently motivated to work the next day; increasing the amount of reward resulted in an animal that would not work for water the following day. A typical session lasted about 20-30 min, during which time a rat received about 75 silent trials interspersed among over 200 sound trials.

The performance of a rat on the silent trials was scored as the percentage of silent trials on which it responded to the left side. Thus, for example, a score of $80 \%$ indicated that a rat had a left preference on the silent trials, whereas a score of $30 \%$ indicated that it had a right preference. We have found that approximately half of the animals prefer the left and the other half the right side, with the degree of the preference varying between individuals but remaining stable. To ensure that the animals' had adapted to the procedure and that their performance was stable, they were trained for 70 sessions before being exposed to a loud tone to induce tinnitus.

Following exposure to a loud tone, a rat's score on the silent trials for a session was compared with its scores on silent trials for the five sessions preceding exposure. This was done by calculating a $z$ score for each session, using the mean and standard deviation of an animal's five preceding preexposure sessions. A positive score for tinnitus was defined as a $z$ score equal to or greater than the .01 one-tailed criterion for the preexposure scores (i.e., $M+S D * 2.33$ ).
Inducing tinnitus

To induce tinnitus, a rat was exposed to a loud sound in the ear opposite the side that it preferred on silent trials. The rat was lightly anesthetized ( $2 \%$ halothane with a 1:1 mixture of nitrous oxide and oxygen), and a foam earplug was placed in its nonexposed ear, with the pinna taped over the meatus to protect it from the sound. The rat was then placed on its side with the nonexposed ear on a pad. The exposing sound was produced by a digital signal generator (Model 3525, Zonic), amplified (Model MPA, 100-W/channel, Radio Shack), and sent to a piezoelectric loudspeaker (Motorola KSN 1005A) or a midrange driver (ElectroVoice $1823 \mathrm{M})$. A plastic funnel with a 4-mm inner diameter tip was attached to the loudspeaker with thermoplastic adhesive, and the tip of the funnel was placed $2 \mathrm{~mm}$ from the concha. The sound was measured with the 1/4-in. microphone placed $2 \mathrm{~mm}$ from the tip of the funnel. The animal was closely observed for the duration of the exposure to ensure that the funnel tip remained in place. The anesthesia was turned off a few minutes before the exposure ended, so that the animal was becoming conscious by the time the exposure was complete. This allowed behavioral testing to begin within $10 \mathrm{~min}$ following the exposure.

The exposing tones consisted of nine different frequencies that were presented at a level of $110 \mathrm{~dB}$ SPL for $10 \mathrm{~min}$ (the level of each tone above the average rat threshold is shown in Table 1). Each animal was exposed to each of the tones, so that the degree to which the tones produced tinnitus could be compared within and between animals. Because the absolute thresholds of humans exposed to similar levels of sound return to normal within $24-48 \mathrm{~h}$ (Davis et al., 1950), allowing at least 8 days between exposures seemed sufficient to minimize interactions between exposures.

\section{Results}

The following sections describe the results of the anesthesia control tests, tone exposures, and simulated tinnitus tests. The order in which the rats were exposed to the different tones and whether or not they tested positive for tinnitus are shown in Table 2.

Anesthesia control test conducted on unexposed rats

Figure 1 illustrates the performance of 7 rats on the silent trials before and after being given a sham exposure that was identical in all respects to the real exposures, with the exception that the exposing tone was not turned on. Three points can be drawn from these results. First, anesthetizing previously unexposed animals does not affect their scores 
Table 1 Tones used to induce tinnitus

\begin{tabular}{lll}
\hline Frequency (in dB SPL) & Rat Threshold $^{*}$ & Intensity Above Threshold (in dB) \\
\hline $1 \mathrm{kHz}$ & 24 & 86 \\
$2 \mathrm{kHz}$ & 22 & 88 \\
$2.5 \mathrm{kHz}$ & 23 & 87 \\
$4 \mathrm{kHz}$ & 13 & 97 \\
$8 \mathrm{kHz}$ & 0 & 110 \\
$16 \mathrm{kHz}$ & 11 & 99 \\
$24 \mathrm{kHz}$ & 4 & 106 \\
$32 \mathrm{kHz}$ & -1 & 111 \\
$45 \mathrm{kHz}$ & 18 & 92 \\
\hline
\end{tabular}

All exposures were of $110 \mathrm{~dB}$ sound pressure level (SPL) for 10 min. * From Heffner et al. (1994)

on silent trials, even though testing was begun within $10 \mathrm{~min}$ after the anesthesia was discontinued. Second, the scores on the silent trials are relatively stable, generally remaining within one standard deviation of the baseline mean over the 23-day test period. Finally, it may be noted that these results do not entirely rule out the possibility that the anesthesia might cause tinnitus, because the test used here was designed to detect monaural tinnitus and might not detect tinnitus that is not lateralized to one side.

Effect of exposure to loud tones

Figures 2 and 3 illustrate the performance of 6 rats on the silent trials before and after exposure to a loud tone $(110 \mathrm{~dB}$ SPL for $10 \mathrm{~min}$ ). As can be seen, scores that were positive for tinnitus generally occurred on the day of the exposure (behavioral testing was begun within 10 min following the exposure). In addition, some animals did test positive on subsequent days, indicating that they still had tinnitus.

Exposure to frequencies from 2 to $24 \mathrm{kHz}$ was more likely to result in a positive score than were exposures to higher and lower frequencies (Fig. 4). There does not appear to be any simple relationship between the sensation level (SL) of the exposing tone (its level above threshold) and the degree to which a tone causes tinnitus, since $32 \mathrm{kHz}$ presented at $111 \mathrm{~dB}$ SL was less likely to induce tinnitus than $2 \mathrm{kHz}$ presented at $88 \mathrm{~dB}$ SL (cf. Table 1 and Fig. 4). Instead, it appears that exposure to frequencies toward the high- and low-frequency ends of an animal's hearing range may be less likely to cause tinnitus than those in the midrange.

Effect of anesthesia on previously exposed animals

The 6 rats used in the tone exposure tests were given three sham exposures (anesthetized, but not exposed to sound). The first sham exposure was given after they had been exposed to seven tones, with the last two sham exposures given after they had been exposed twice more to loud tones (55 and 61 days after the first sham exposure, respectively). As can be seen in Table 2 and Fig. 5, 2 of the rats never tested positive (B and $C), 1$ tested positive once $(F)$, and 3 tested positive twice (A, D, and E); in short, the sham trials yielded a positive score for tinnitus about one out of three times. Because the unexposed rats never tested positive for lateralized tinnitus (Fig. 1), this result suggests that the
Table 2 Order in which the exposing tones, anesthesia controls, and simulated tinnitus tests were given for each rat and whether or not an animal tested positive for tinnitus
* Indicates that an animal tested positive on the day of the exposure, anesthesia, or the first day of the simulated tinnitus test using the .01 , one-tailed level criterion

\begin{tabular}{|c|c|c|c|c|c|}
\hline Rat A & Rat B & Rat C & Rat D & Rat E & Rat F \\
\hline $8 \mathrm{kHz}$ & $4 \mathrm{kHz}{ }^{*}$ & $2 \mathrm{kHz}$ & $16 \mathrm{kHz}^{*}$ & $32 \mathrm{kHz}$ & $45 \mathrm{kHz}$ \\
\hline $2 \mathrm{kHz}{ }^{*}$ & $32 \mathrm{kHz}$ & $16 \mathrm{kHz}^{*}$ & $45 \mathrm{kHz}$ & $4 \mathrm{kHz}^{*}$ & $8 \mathrm{kHz}^{*}$ \\
\hline $16 \mathrm{kHz}^{*}$ & $8 \mathrm{kHz}{ }^{*}$ & $45 \mathrm{kHz}$ & $4 \mathrm{kHz}^{*}$ & $2 \mathrm{kHz}^{*}$ & $32 \mathrm{kHz}$ \\
\hline $32 \mathrm{kHz}^{*}$ & $2 \mathrm{kHz}$ & $32 \mathrm{kHz}$ & $2 \mathrm{kHz}^{*}$ & $24 \mathrm{kHz}^{*}$ & $2 \mathrm{kHz}^{*}$ \\
\hline $24 \mathrm{kHz}^{*}$ & $24 \mathrm{kHz}$ & $24 \mathrm{kHz}^{*}$ & $24 \mathrm{kHz}^{*}$ & $2.5 \mathrm{kHz}^{*}$ & $24 \mathrm{kHz}^{*}$ \\
\hline $2.5 \mathrm{kHz}^{*}$ & $2.5 \mathrm{kHz}$ & $2.5 \mathrm{kHz}^{*}$ & $2.5 \mathrm{kHz}^{*}$ & $8 \mathrm{kHz}^{*}$ & $2.5 \mathrm{kHz}^{*}$ \\
\hline $4 \mathrm{kHz}^{*}$ & $16 \mathrm{kHz}^{*}$ & $8 \mathrm{kHz}^{*}$ & $8 \mathrm{kHz}^{*}$ & $16 \mathrm{kHz}^{*}$ & $16 \mathrm{kHz}^{*}$ \\
\hline Anesthesia ${ }^{*}$ & Anesthesia & Anesthesia & Anesthesia* & Anesthesia ${ }^{*}$ & Anesthesia \\
\hline $45 \mathrm{kHz}^{*}$ & $45 \mathrm{kHz}$ & $4 \mathrm{kHz}^{*}$ & $32 \mathrm{kHz}$ & $45 \mathrm{kHz}^{*}$ & $4 \mathrm{kHz}^{*}$ \\
\hline $1 \mathrm{kHz}$ & $1 \mathrm{kHz}$ & $1 \mathrm{kHz}{ }^{*}$ & $1 \mathrm{kHz}$ & $1 \mathrm{kHz}^{*}$ & $1 \mathrm{kHz}$ \\
\hline Simulated ${ }^{*}$ & Simulated ${ }^{*}$ & Simulated & Simulated & Simulated & Simulated ${ }^{*}$ \\
\hline Anesthesia ${ }^{*}$ & Anesthesia & Anesthesia & Anesthesia & Anesthesia & Anesthesia \\
\hline Anesthesia & Anesthesia & Anesthesia & Anesthesia ${ }^{*}$ & Anesthesia ${ }^{*}$ & Anesthesia ${ }^{*}$ \\
\hline
\end{tabular}




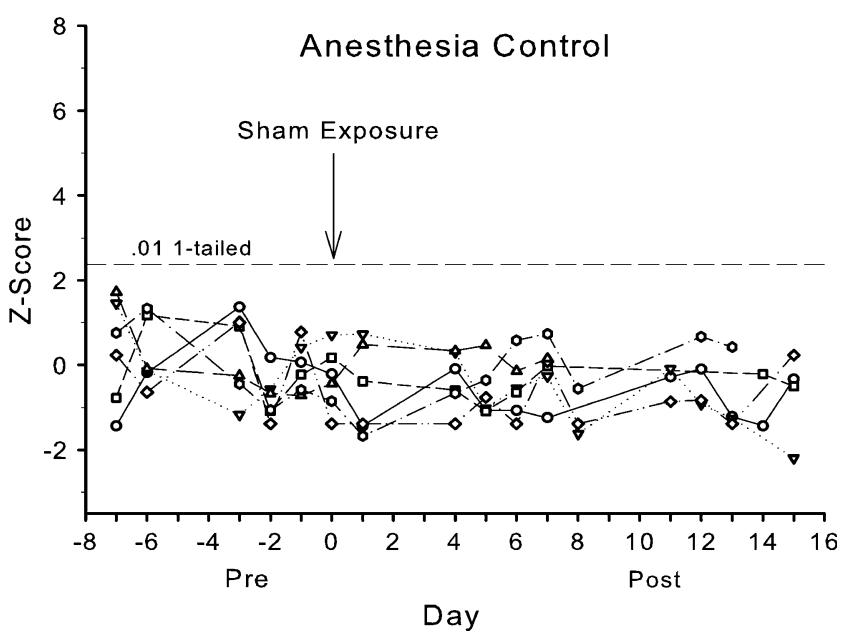

Fig. 1 Performance of 7 rats on the silent trials before and after receiving a 10-min sham exposure under halothane/nitrous oxide anesthesia. The animals had not been previously exposed to loud sound. The side preferences of the rats on the silent trials tended to increase over time, with no indication of a shift toward the less-preferred side. The $z$ scores were computed using the mean and standard deviation of the five sessions preceding the sham exposure. The horizontal dashed line indicates the .01 , one-tailed criterion level

halothane/nitrous oxide anesthesia may have reinstated tinnitus in animals that had previously had it. Thus, it is possible that, after the first exposure, some of the positive scores in Figs. 2 and 3 were due to the anesthesia.

\section{Simulated tinnitus}

The effect of monaural tinnitus was simulated by continuously presenting a $16-\mathrm{kHz}$ band of noise at $25 \mathrm{~dB}$ above the rat threshold. The sound was presented from a loudspeaker located on the side of the animal opposite the side it normally preferred on the silent trials. The results showed that, although all 6 of the animals shifted their responding on the silent trials on the first day, 2 of them (Rats D and F) failed to respond to it on one or more of the subsequent sessions (Fig. 6). This failure to shift on some sessions may reflect habituation to the simulated tinnitus.

\section{Hearing loss}

As noted in the Method section, the level and duration of the exposing tones ( $110 \mathrm{~dB}$ for $10 \mathrm{~min}$ ) was chosen because the absolute thresholds of humans exposed to such tones return to normal levels within $24-48 \mathrm{~h}$ (Davis et al., 1950). However, due to time constraints, it was not possible to determine the rats' threshold shifts using behavioral tests, and physiological measures of sensorineural hearing loss, such as the auditory brainstem response, are not sufficiently reliable to be useful (Heffner, Koay, \& Heffner, 2008). However, because a sudden unilateral hearing loss will disrupt sound localization performance, an indication of the resulting hearing loss can be obtained from an animal's performance on the sound trials.

Table 3 shows the number of days following exposure before an animal's score on the sound trials returned to a level within the range of the five sessions preceding the exposure. As this table shows, the most common occurrence (38 out of 54 instances) was for an animal's score on the sound trials to drop on the day of the exposure and to return to preexposure levels on the following day (indicated by a 1 in Table 3). In 9 instances, the exposure did not noticeably affect performance (indicated by 0 ), whereas in the remaining 7 instances, recovery to preexposure levels did not occur until 2 days after exposure (indicated by a 2). In this respect, the recovery time for the rats' thresholds is similar to that of humans exposed to the same SPLs (Davis et al., 1950). Although these results do not prove that the animals had no residual hearing loss, they do demonstrate that any such loss had no noticeable effect on their sound localization performance. With regard to the silent trials, background sound that could have biased the animals on the silent trials to the side of the unexposed ear was eliminated by the double-walled sound chamber that prevented outside sounds from entering the chamber and by using equipment within the chamber did not produce any detectable sound.

\section{Discussion}

The two-choice sound localization test provides a conceptually straightforward test of lateralized tinnitus in animals. One of the strengths of this test is that each animal serves as its own control, making it possible to assess tinnitus in individual animals. However, no test is without its limitations, and the following discussion explores the accuracy and reliability of this test. (For brief descriptions of other animal tinnitus tests, see the reviews by Bauer \& Brozoski, 2008, and Turner, 2007.)

Accuracy of the two-choice procedure in detecting tinnitus

The first requirement of any diagnostic test is accuracythat is, it should correctly distinguish individuals that have a particular disorder from those that do not. The anesthesia control test addressed the question of whether an animal that has not been exposed to a loud sound will test positive for tinnitus. As shown in Fig. 1, none of the 7 rats tested positive on the day of the sham exposure, nor did their side preference on the silent trials vary significantly during the following 2 weeks. Thus, the possibility of a false positive result for this test appears quite low.

The probability that an animal with tinnitus would test positive on the two-choice test was addressed in the simulated tinnitus test, in which a low-level (25 dB SL), 

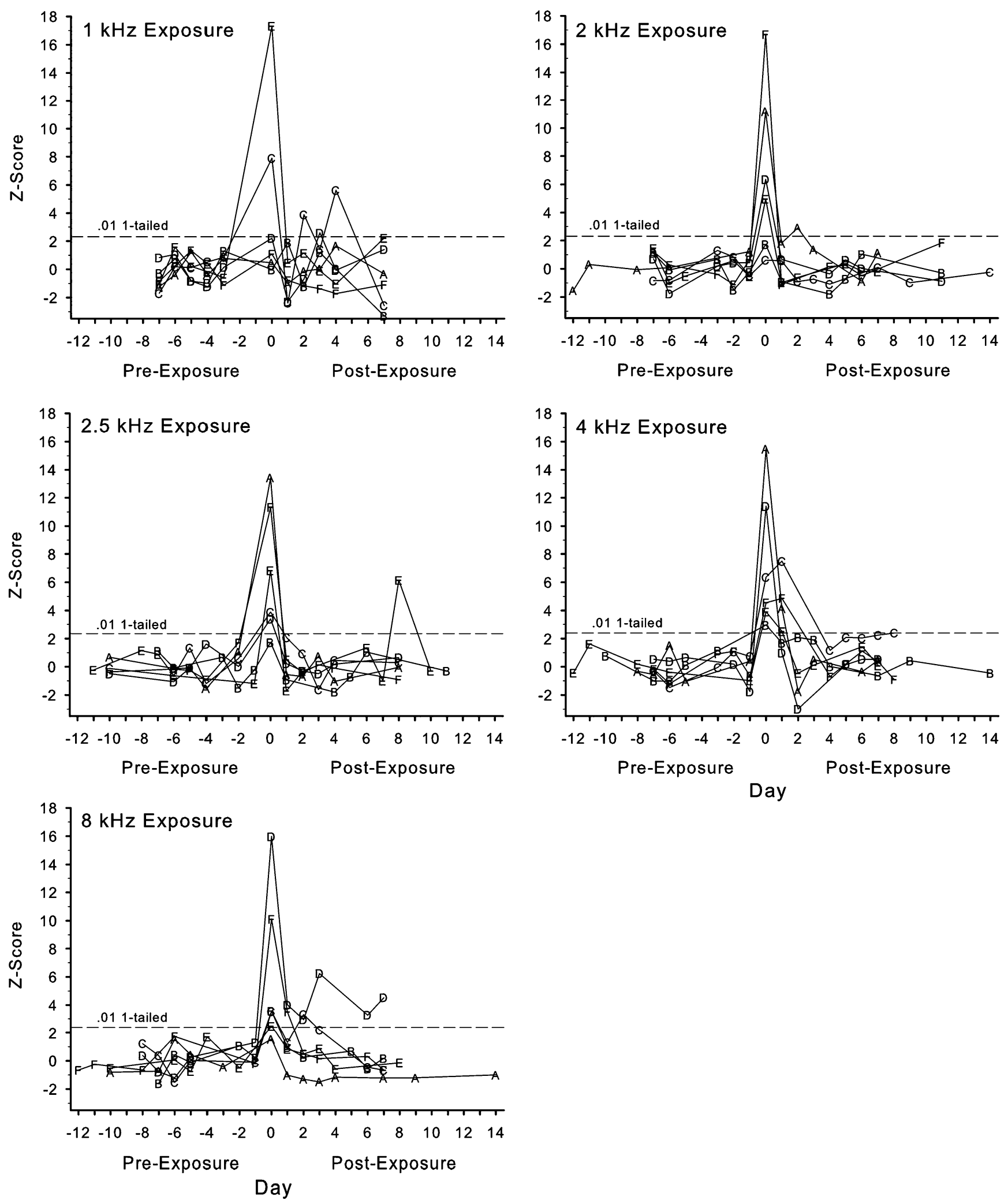

Fig. 2 Performance of 6 rats (A-F) on the silent trials before and after receiving a 10-min, 110-dB SPL exposure to five different frequencies. The horizontal dashed line indicates the .01 , one-tailed criterion level 

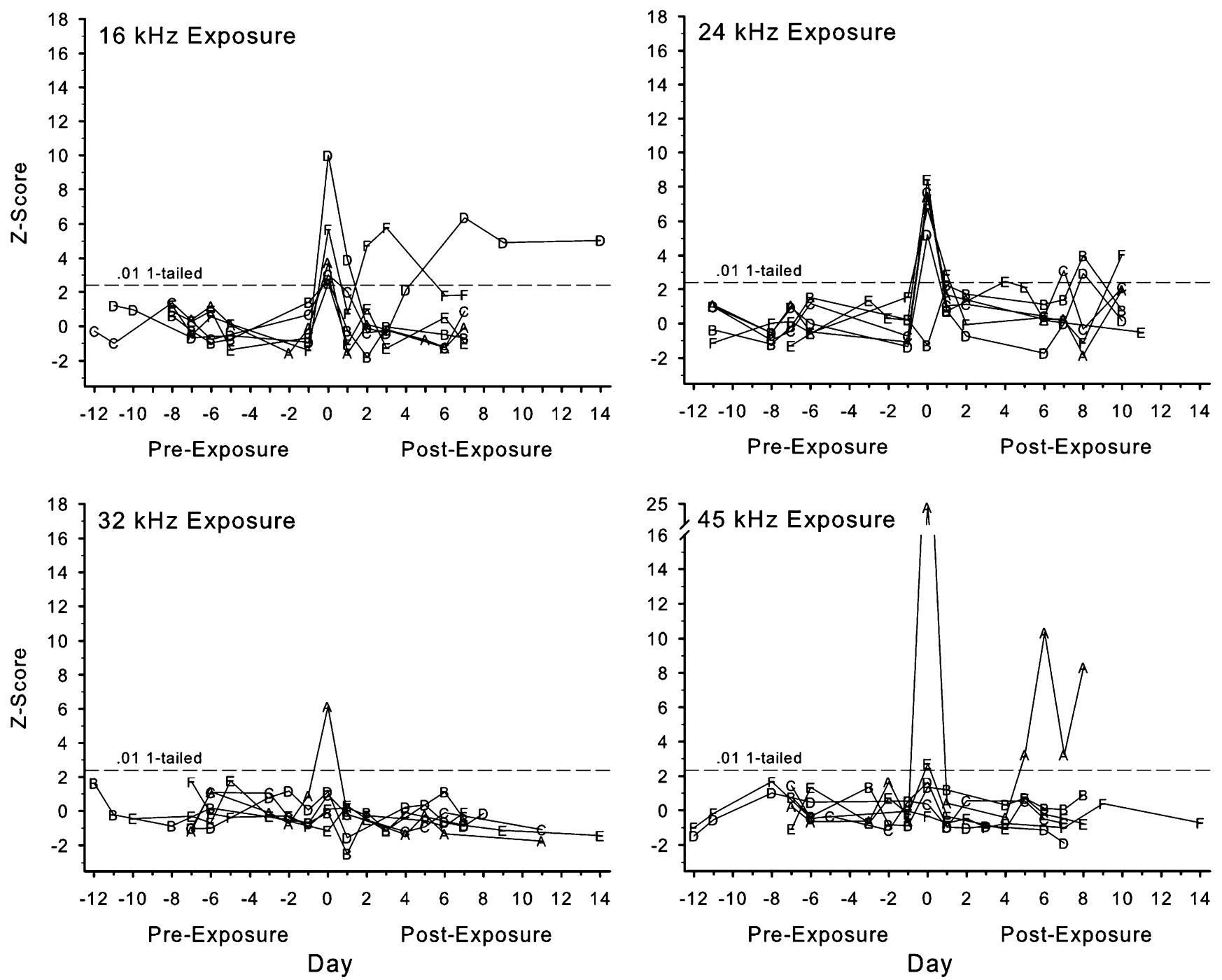

Fig. 3 Performance of 6 rats (labeled A-F) on the silent trials before and after receiving a $10-\mathrm{min}, 110-\mathrm{dB}$ SPL exposure to four different frequencies. The horizontal dashed line indicates the .01 , one-tailed

$16-\mathrm{kHz}, 1 / 3$-octave band noise was continuously presented on one side. As shown in Fig. 6, all 7 of the rats tested positive on the first day, indicating that the test is $100 \%$ effective in detecting the onset of simulated tinnitus, at least tinnitus that has a salience to an animal that is equal to or greater than the 25-dB SL noise used here. However, 2 of the rats (D and F) did show some adaptation to the $16-\mathrm{kHz}$ noise, and failed to test positive on some subsequent days, indicating that after the first day, the detection rate of this test drops to $84 \%$. However, it may be possible to increase the success rate by training animals prior to exposure to respond to simulated tinnitus for longer periods of time prior to exposure and by selecting animals that respond reliably to simulated tinnitus over long periods of time.

Finally, for this procedure to work as described, the tinnitus must be lateralized to one side or the other. As a result, it may not work well in cases of bilateral tinnitus, such criterion level. Note the break in the vertical axis of the $45-\mathrm{kHz}$ exposure, which was done to accommodate the Day $0 z$ score of 24.7 for Rat A

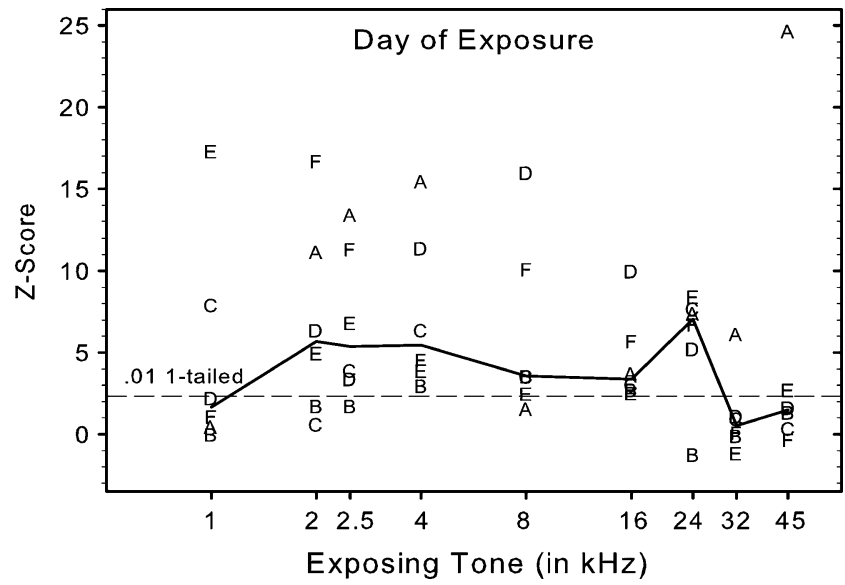

Fig. 4 The $z$ scores of 6 rats for the day of exposure to the nine frequencies. The solid line indicates the median score for each frequency. The horizontal dashed line indicates the .01 , one-tailed criterion level 

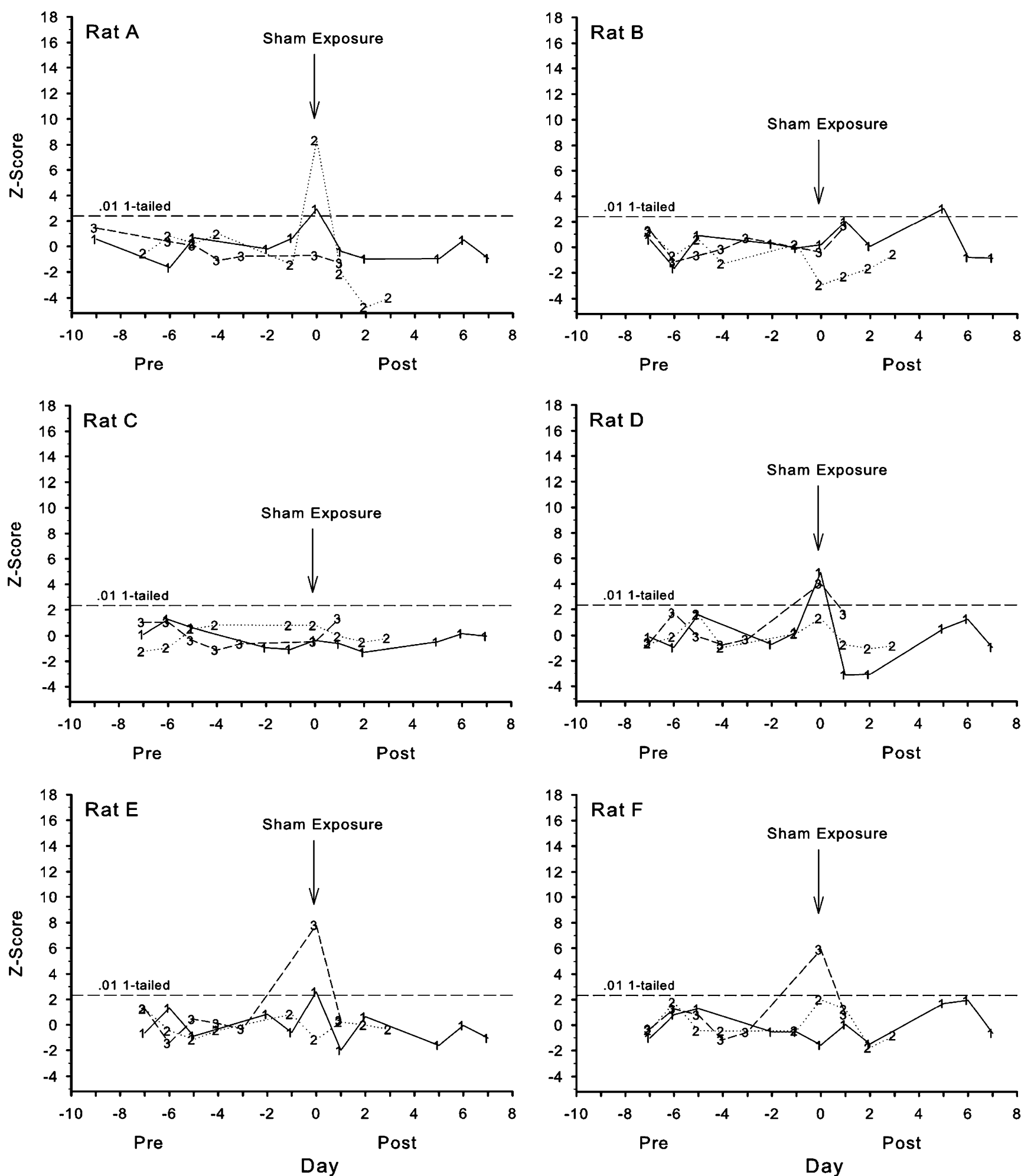

Fig. 5 Effect of the halothane/nitrous oxide anesthesia on rats that had previously tested positive for tinnitus. A comparison of these results with those of Fig. 1 suggests that although the anesthesia itself does not cause tinnitus, it can reinstate tinnitus in animals that have

previously tested positive for it. The numbers plotted in each graph indicate the first, second, and third anesthesia/sham exposure (see Table 2). The horizontal dashed line indicates the .01, one-tailed criterion level 

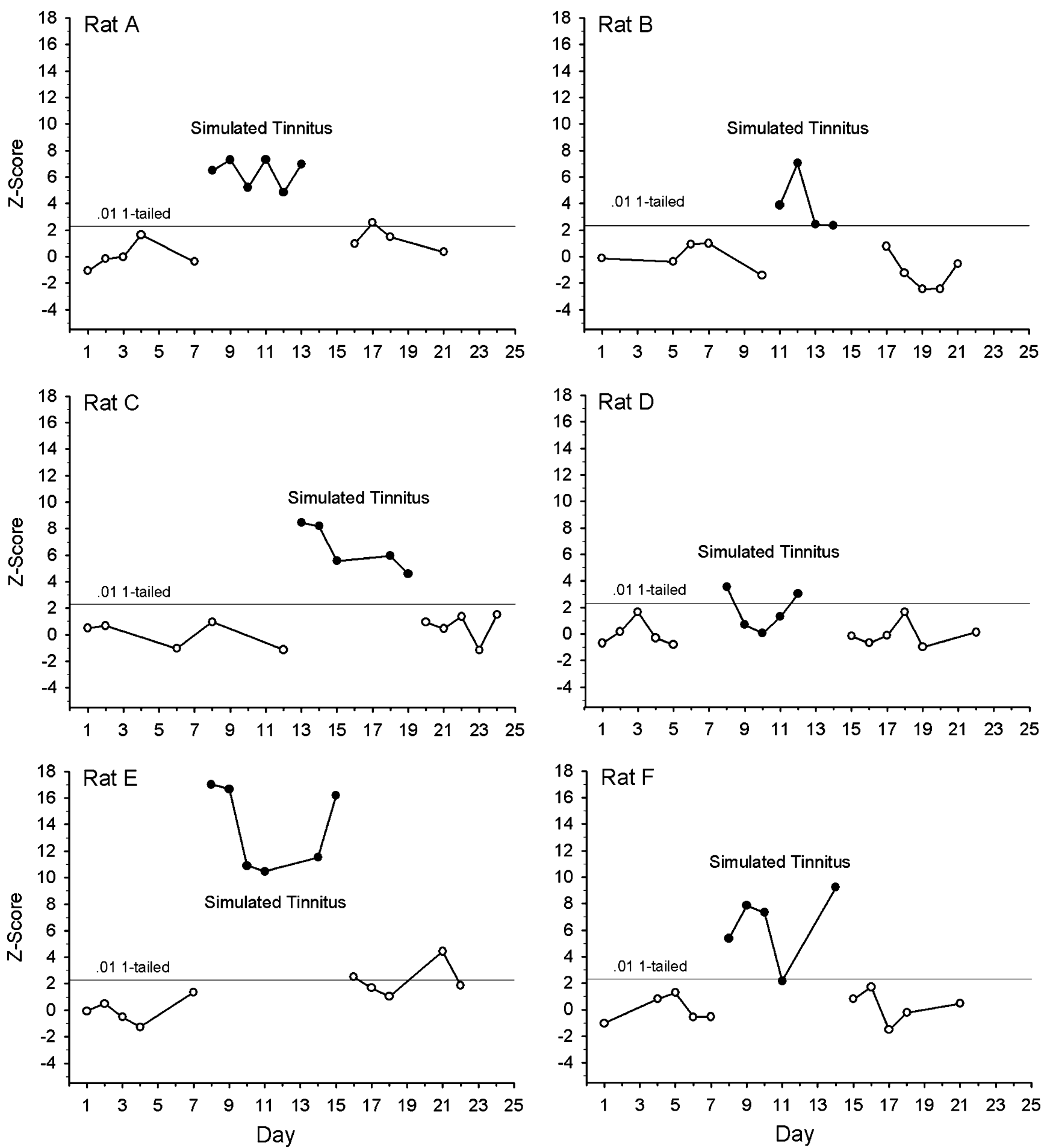

Fig. 6 Effect of simulating tinnitus by presenting continuous $16-\mathrm{kHz}$ narrow-band noise at $25 \mathrm{~dB}$ above the rat threshold on the side of an animal opposite the one to which it normally responded on the silent trials

as that induced by salicylate or other chemicals. However, since it is well established that tinnitus caused by exposing one ear to a loud sound is always lateralized to the exposed ear (Atherley et al., 1968; Davis et al., 1950; Loeb \& Smith, 1967), this procedure should work well for tinnitus caused by overstimulating or otherwise damaging one ear.

\section{Effect of anesthesia}

The effect of anesthesia proved interesting, because it caused some of the previously exposed rats to test positive for lateralized tinnitus (Fig. 5), whereas none of the unexposed animals tested positive (Fig. 1). As previously 
Table 3 Number of days following exposure in which the scores on the sound trials fell below the lowest score of the previous five sessions, indicating the duration of a transient unilateral hearing loss

\begin{tabular}{lllllll}
\hline Frequency & Rat A & Rat B & Rat C & Rat D & Rat E & Rat F \\
\hline $1 \mathrm{kHz}$ & 1 & 0 & 1 & 0 & 2 & 1 \\
$2 \mathrm{kHz}$ & 1 & 1 & 1 & 1 & 2 & 1 \\
$2.5 \mathrm{kHz}$ & 1 & 1 & 1 & 1 & 2 & 1 \\
$4 \mathrm{kHz}$ & 1 & 1 & 2 & 1 & 1 & 2 \\
$8 \mathrm{kHz}$ & 1 & 1 & 1 & 1 & 2 & 1 \\
$16 \mathrm{kHz}$ & 1 & 1 & 1 & 1 & 0 & 0 \\
$24 \mathrm{kHz}$ & 1 & 0 & 1 & 1 & 1 & 2 \\
$32 \mathrm{kHz}$ & 1 & 1 & 1 & 1 & 0 & 0 \\
$45 \mathrm{kHz}$ & 1 & 0 & 1 & 0 & 1 & 1 \\
\hline
\end{tabular}

noted, we cannot rule out the possibility that some of the unexposed animals might have developed tinnitus that did not affect their responding, because it was not lateralized. Nevertheless, these results suggest that the anesthesia was not the primary cause of the tinnitus, but rather was reinstating it.

Although the anesthesia control tests suggest that up to a third of the positive scores could be due to the anesthesia, there are two reasons why the actual number is probably lower. First, the effect of the anesthesia would not be expected to occur for the initial tone exposures, and thus the first positive score for each animal is unlikely to have been the result of the anesthesia. Second, there would be instances in which both the tone and the anesthesia were sufficient to produce tinnitus.

In humans, general anesthesia is not considered to produce transient tinnitus (Schaaf, Kampe, \& Hesse, 2004), possibly because, as the results here suggest, most individuals will not experience it, and it will be gone within $24 \mathrm{~h}$ in those that do. With regard to animal research, this finding has several implications. First, to avoid the possibility of anesthesia reinstating tinnitus, subsequent exposures to loud sound should be made with the animal restrained but not anesthetized. Second, the fact that anesthesia can reinstate tinnitus is itself of interest. For example, do anesthetics vary in their ability to reinstate tinnitus, and if so, what are the mechanisms underlying the differences? Finally, other events might be able to reinstate tinnitus, such as a brief exposure to a loud sound that, by itself, would not be expected to cause tinnitus; indeed, to avoid such a possibility, the metal lid of the animal box in which the rats in this study were weighed and transported was padded, to avoid producing the loud sounds that can occur when the lid is placed on or removed from the box.
Effect of frequency of the exposing tone

Although the order in which the rats were exposed to the different tones was not sufficiently randomized to permit a detailed comparison, it does appear that some tones were less likely to cause tinnitus than others. Whereas at least 4 of the 6 rats tested positive for tinnitus when exposed to frequencies from 2 to $24 \mathrm{kHz}$, exposure to 1 and $45 \mathrm{kHz}$ caused only 2 of the 6 animals to test positive, and exposure to $32 \mathrm{kHz}$ resulted in only one positive score (see Fig. 4 and Table 2). As previously noted, this result does not seem related to SL; for example, $32 \mathrm{kHz}$, which was presented at $111 \mathrm{~dB}$ SL, was less likely to induce tinnitus than $2 \mathrm{kHz}$, which was presented at $88 \mathrm{~dB}$ SL. Nor can it be easily attributed to the order in which the rats were exposed, in that some positive scores might result from the anesthesia reinstating tinnitus. This is because the $45-\mathrm{kHz}$ exposures came relatively late in the series, and the $1-\mathrm{kHz}$ exposure was the last one given; although the $32-\mathrm{kHz}$ exposures were given relatively early in the sequence, the $2-\mathrm{kHz}$ exposures were made, on average, even earlier, and they resulted in four positive scores.

Given that the highest and lowest frequencies audible to the Norway rat at a level of $60 \mathrm{~dB}$ SPL are 0.530 and $68 \mathrm{kHz}$ (Heffner et al., 1994), it appears that frequencies within an octave of an animal's 60-dB low- and high-frequency hearing limits are less likely to produce tinnitus than frequencies in the midrange. Whether one or another midrange frequency is more likely to produce tinnitus is not yet clear.

\section{Duration of tinnitus}

For the 36 occasions on which a rat tested positive for tinnitus immediately after exposure, the animal tested positive on a subsequent day 17 times. Of these 17 instances, 11 were cases in which the rat tested positive, then negative, and then positive again. A good example of this pattern of responding is the $45-\mathrm{kHz}$ exposure of Rat A (Fig. 3). Because Rats D and F showed a similar pattern of responding in the simulated tinnitus test (Fig. 6), it is possible that the tinnitus was not the element that varied, but the response of the animals to the tinnitus. One way to improve the ability of the two-choice test to follow tinnitus would be to use the simulated tinnitus test to select animals that do not habituate to the external stimulus. Another would be to train animals by occasionally rewarding them for going to the side of the simulated tinnitus prior to inducing tinnitus.

Salience of the resulting tinnitus

As can be seen in Figs. 2, 3 and 4, the degree of an animal's shift to the side of the exposed ear varied greatly, and it may well indicate the salience of tinnitus. For example, Rat 
A's tinnitus following its exposure to $45 \mathrm{kHz}$ may have been louder than that following its exposure at $32 \mathrm{kHz}$. This relationship could be explored and quantified by giving animals simulated tinnitus tests using a range of frequencies and intensities.

\section{Tinnitus and stress}

Because stress has been implicated as an aggravating factor, or even a cause, of tinnitus (see, e.g., Knipper, Zimmermann, \& Müller, 2010), the question arises as to whether the procedures used here may have increased an animal's level of stress. One potential source of stress is water deprivation, which increases the levels of hormones and proteins (c-Fos) that are associated with stress (Arnhold, Wotus, \& Engeland, 2007; Jørgensen, Knigge, Kjær, \& Warberg, 2002). However, most studies have only looked at the initial effects of restricting an animal's water intake (e.g., 1 or 6 days as in the previously cited studies), and a longer-term study found that hormonal levels in rats on water restriction had returned to normal by 8 weeks (Sakellaris \& Vernikos-Danellis, 1974). Given that the rats in the present study were on water restriction for 14 weeks before being exposed to loud sound, they had time to become adapted to the procedure.

A second potential source of stress is the shock that the animals received when they made an error on the sound trials (the use of shock was necessitated by our observation that rats do not perform well on a two-choice sound localization task if errors are not punished). Although the shock was individually adjusted for each rat to the lowest level that would maintain good performance, it would have to cause some stress to be effective. As with water restriction, the animals had 14 weeks to become adapted to the procedure.

Finally, it can be argued that the light anesthesia used to immobilize the rats during the monaural exposure to loud sound was stressful. Indeed, it is possible that the occasional reinstatement of tinnitus in some rats following anesthesia was due to the stress of being anesthetized rather than being a direct effect of the anesthesia itself. As previously noted, animals could be exposed to loud sound without anesthesia by restraining them, although they would have to be acclimated to this, because restraint itself can cause stress (e.g., Jørgensen et al., 2002) - and they would then hear the loud sound, which would also be stressful.

In short, as with an animal's life in the wild, it is virtually impossible to eliminate all potential stressors, especially in tasks that require vigilance and effort, although stress can be minimized. For example, if water restriction proves to be more stressful than food restriction, a food reward can easily be substituted for the water reward. However, a better understanding of the role of stress on tinnitus awaits studies of its effect in humans, because current knowledge is based primarily on clinical observations as opposed to empirical studies (e.g., Hébert \& Lupien, 2007).

Author note I thank K. Marchetto for conducting the behavior tests and R. Heffner and G. Koay for their comments on an earlier draft of the manuscript. Also greatly appreciated were the comments of an informed and diligent reviewer. Supported by NIH Grant DC03258.

\section{References}

Arnhold, M. M., Wotus, C., \& Engeland, W. C. (2007). Differential regulation of parvocellular neuronal activity in the paraventricular nucleus of the hypothalamus following single vs. repeated episodes of water restriction-induced drinking. Experimental Neurology, 206, 126-136. doi:10.1016/j.expneurol.2007.04.010

Atherley, G. R. C., Hempstock, T. I., \& Noble, W. G. (1968). Study of tinnitus induced temporarily by noise. Journal of the Acoustical Society of America, 44, 1503-1506. doi:10.1121/1.1911288

Bauer, C. A., \& Brozoski, T. J. (2008). Tinnitus: Theories, mechanisms, and treatments. In J. Schacht, A. N. Popper, \& R. R. Fay (Eds.), Auditory trauma, protection, and repair (pp. 101129). New York: Springer.

Bauer, C. A., Brozoski, T. J., Rojas, R., Boley, J., \& Wyder, M. (1999). Behavioral model of chronic tinnitus in rats. Otolaryngology-Head and Neck Surgery, 121, 457-462. doi:10.1016/S0194-5998(99) 70237-8

Davis, H., Morgan, C. T., Hawkins, J. E., Jr., Galambos, R., \& Smith, F. W. (1950). Temporary deafness following exposure to loud tones and noise. Acta Oto-Laryngologica, 88(Suppl), 1-57.

Gellermann, L. W. (1933). Chance orders of alternating stimuli in visual discrimination experiments. Journal of Genetic Psychology, 42, 206-208.

Guitton, M. J., Caston, J., Ruel, J., Johnson, R. M., Pujol, R., \& Puel, J.-L. (2003). Salicylate induces tinnitus through activation of cochlear NMDA receptors. Journal of Neuroscience, 23, 3944-3952.

Guitton, M. J., \& Dudai, Y. (2007). Blockade of cochlear NMDA receptors prevents long-term tinnitus during a brief consolidation window after acoustic trauma. Neural Plasticity, 80904, 1-11. doi:10.1155/2007/80904

Hébert, S., \& Lupien, S. J. (2007). The sound of stress: Blunted cortisol reactivity to psychosocial stress in tinnitus sufferers. Neuroscience Letters, 411, 138-142. doi:10.1016/j.neulet.2006.10.028

Heffner, H. E., \& Harrington, I. A. (2002). Tinnitus in hamsters following exposure to intense sound. Hearing Research, 170, 83-95. doi:10.1016/S0378-5955(02)00343-X

Heffner, H. E., Heffner, R. S., Contos, C., \& Ott, T. (1994). Audiogram of the hooded Norway rat. Hearing Research, 73, 244-247. doi:10.1016/0378-5955(94)90240-2

Heffner, H. E., \& Koay, G. (2005). Tinnitus and hearing loss in hamsters (Mesocricetus auratus) exposed to loud sound. Behavioral Neuroscience, 119, 734-742. doi:10.1037/0735-7044.119.3.734

Heffner, H. E., Koay, G., \& Heffner, R. S. (2008). Comparison of behavioral and auditory brainstem response measures of threshold shift in rats exposed to loud sound. Journal of the Acoustical Society of America, 124, 1093-1104. doi:10.1121/1.2949518

Jastreboff, P. J., Brennan, J. F., Coleman, J. K., \& Sasaki, C. T. (1988). Phantom auditory sensation in rats: An animal model for tinnitus. Behavioral Neuroscience, 102, 811-822. doi:10.1037/07357044.102.6.811 
Jørgensen, H., Knigge, U., Kjær, A., \& Warberg, J. (2002). Serotonergic involvement in stress-inducted vasopressin and oxytocin secretion. European Journal of Endocrinology, 147, 815-824. doi:10.1530/eje.0.1470815

Kizawa, K., Kitahara, T., Horii, A., Maekawa, C., Kuramasu, T., Kawashima, T., et al. (2010). Behavioral assessment and identification of a molecular marker in a salicylate-induced tinnitus in rats. Neuroscience, 165, 1321-1332. doi:10.1016/j. neuroscience.2009.11.048

Knipper, M., Zimmermann, U., \& Müller, M. (2010). Molecular aspects of tinnitus. Hearing Research, 266, 60-69. doi:10.1016/j. heares.2009.07.013

Lobarinas, E., Sun, W., Cushing, R., \& Salvi, R. (2004). A novel behavioral paradigm for assessing tinnitus using scheduleinduced polydipsia avoidance conditioning (SIP-AC). Hearing Research, 190, 109-114. doi:10.1016/S0378-5955(04)00019-X

Loeb, M., \& Smith, R. P. (1967). Relation of inducted tinnitus to physical characteristics of the inducing stimuli. Journal of the Acoustical Society of America, 42, 453-455. doi:10.1121/ 1.1910600

Martines, F., Bentivegna, D., Di Piazza, F., Martines, E., Sciacca, V., \& Martinciglio, G. (2010). Investigation of tinnitus patients in Italy: Clinical and audiological characteristics. International Journal of Otolaryngology, 265861, 1-8. doi:10.1155/2010/ 265861

Rűttiger, L., Ciuffani, J., Zenner, H.-P., \& Knipper, M. (2003). A behavioral paradigm to judge acute sodium salicylate-induced sound experience in rats: A new approach for an animal model on tinnitus. Hearing Research, 180, 39-50. doi:10.1016/S03785955(03)00075-3

Rybalko, N., \& Syka, J. (2005). Effect of noise exposure on gap detection in rats. Hearing Research, 200, 63-72. doi:10.1016/j. heares.2004.08.014

Sakellaris, P. C., \& Vernikos-Danellis, J. (1974). Alteration of pituitary-adrenal dynamics inducted by a water deprivation regimen. Physiology and Behavior, 12, 1067-1070. doi:10.1016/ 0031-9384(74)90156-5

Schaaf, H., Kampe, S., \& Hesse, G. (2004). Tinnitus nach Anästhesie. Anaesthesist, 53, 358-361. doi:10.1007/s00101-004-0667-3

Turner, J. G. (2007). Behavioral measures of tinnitus in laboratory animals. Progress in Brain Research, 166, 147-156. doi:10.1016/ S0079-6123(07)66013-0

Turner, J. G., Brozoski, T. J., Bauer, C. A., Parrish, J. L., Myers, K., Hughes, L. F., et al. (2006). Gap detection deficits in rats with tinnitus: A potential novel screening tool. Behavioral Neuroscience, 120, 188-195. doi:10.1037/0735-7044.120.1.188

Wesolek, C. M., Koay, G., Heffner, R. S., \& Heffner, H. E. (2010). Laboratory rats (Rattus norvegicus) do not use binaural phase differences to localize sound. Hearing Research, 265, 54-62. doi:10.1016/j.heares.2010.02.011

Zheng, Y., Hooton, K., Smith, P. F., \& Darlington, C. L. (2008). Carbamazepine reduces the behavioural manifestations of tinnitus following salicylate treatment in rats. Acta Oto-Laryngologica, 128, 48-52. doi:10.1080/00016480701361939 